\title{
ON THE BOUNDARY BEHAVIOUR OF UNIVALENT HARMONIC MAPPINGS
}

\section{Daoud Bshouty, Abdallah Lyzzaik and Allen Weitsman}

Technion, Department of Mathematics

Haifa 32000, Israel; daoud@tx.technion.ac.il

American University of Beirut, Department of Mathematics

Beirut, Lebanon; lyzzaik@aub.edu.lb

Purdue University, Department of Mathematics

West Lafayette, IN 47907-1395, U.S.A.; weitsman@purdue.edu

\begin{abstract}
We investigate various boundary behavior properties of harmonic mappings of the unit disk D with emphasis on univalent harmonic mappings "onto" D in the sense of Hengartner and Schober [10]. This study continues the earlier work of the authors [4] on a problem that was raised by Laugesen [12].
\end{abstract}

\section{Introduction}

A harmonic mapping $f$ of a complex region $G$ is a complex-valued function that satisfies Laplace's equation

$$
\Delta f \equiv f_{x x}+f_{y y}=0 .
$$

This function can be written as

$$
f(z)=u(x, y)+i v(x, y), \quad z=x+i y,
$$

where $u$ and $v$ are real-valued harmonic functions, and

$$
f(z)=h(z)+\overline{g(z)}
$$

where $h$ and $g$ are analytic functions which are single-valued if $G$ is simply-connected and possibly multiple-valued if $G$ is otherwise. In the former case, the function $h$ will be called the analytic factor, and $\bar{g}$ the coanalytic factor of $f$, and the function $a=g^{\prime} / h^{\prime}$ the second complex dilatation. Then $|a|<1$ in $G$ if and only if $f$ is open and sense-preserving, and $|a|>1$ in $G$ if and only if $f$ is open and sense-reversing.

Throughout this article, we denote by $\mathbf{C}, \mathbf{D}$, and $\mathbf{T}$ the complex plane, the open unit disc, and the unit circle, respectively.

A way to construct harmonic mappings of $\mathbf{D}$ is as follows. Let $f^{*}\left(e^{i \theta}\right)$ be a Lebesgue integrable function on $\mathbf{T}$. Then

$$
f(z)=P\left[f^{*}\right]=\frac{1}{2 \pi} \int_{0}^{2 \pi} P(r, \varphi-\theta) f^{*}\left(e^{i \varphi}\right) d \varphi, \quad z=r e^{i \theta} \in \mathbf{D},
$$

where $P(r, t)$ is the Poisson kernel of $\mathbf{D}$, is a harmonic mapping of $\mathbf{D}$ whose unrestricted limit at every continuity point $e^{i \theta_{0}}$ of $f^{*}$ is $f^{*}\left(e^{i \theta_{0}}\right)$. Set $f^{*}\left(e^{i \theta}\right) \equiv \Phi(\theta)$; these representations will be used interchangeably wherever it is appropriate.

doi:10.5186/aasfm.2012.3701

2010 Mathematics Subject Classification: Primary 30J99, 31A99.

Key words: Harmonic mapping, analytic dilatation, convex domains, elliptic differential equation. 
The Rado-Kneser-Choquet theorem [8, pp. 29-30] asserts that if $\left|f^{*}\right|=1$ and $\arg f^{*}\left(e^{i \theta}\right)$ is nondecreasing in $\theta \in[0,2 \pi)$ with $\Delta_{\partial D} \arg f^{*}\left(e^{i \theta}\right) \leq 2 \pi$ on $\mathbf{T}$, and $f^{*}$ takes at least three distinct values on $\mathbf{T}$, then $f$ is a univalent sense-preserving harmonic mapping of $\mathbf{D}$ onto a convex region whose "vertices" lie on $\mathbf{T}$.

Open sense-preserving harmonic mappings of $\mathbf{D}$ also arise as solutions of linear elliptic partial differential equations of the form

$$
\overline{f_{\bar{z}}}(z)=a(z) f_{z}(z), \quad z \in \mathbf{D},
$$

where $a$ is an analytic function from $\mathbf{D}$ into itself. Thus with the representation (1),

$$
\left|g^{\prime}\right|<\left|h^{\prime}\right|, \quad z \in D \text {. }
$$

For the special case where $|a|<k<1$ in $\mathbf{D}$, it is classical that the existence part of the Riemann Mapping Theorem (RMT) of equation (3) holds; namely, for a given bounded simply connected domain $\Omega$ and a fixed $w_{0} \in \Omega$, there is a univalent solution $f$ of (3) that satisfies $f(0)=w_{0}$ and $f_{z}(0)>0$ and maps $\mathbf{D}$ onto $\Omega$. In addition, if $\Omega$ is a Jordan domain, then $f$ extends to a homeomorphism from $\overline{\mathbf{D}}$ onto $\bar{\Omega}$. However, in the case where $\|a(z)\|_{\infty}=1$ the following theorem holds [10, Theorem 4.2 and Theorem 4.3].

Theorem A. (Hengartner and Schober [10]) Let $\Omega$ be a bounded simply connected domain whose boundary $\partial \Omega$ is locally connected. Suppose that $a(\mathbf{D}) \subset \mathbf{D}$ and $w_{0}$ is a fixed point of $\Omega$. Then there exists a univalent solution $f$ of (3) having the following properties:

(a) $f(0)=w_{0}, f_{z}(0)>0$, and $f(U) \subset \Omega$.

(b) There is a countable set $E \subset \partial U$ such that the unrestricted limits $f^{*}\left(e^{i t}\right)=$ $\lim _{z \rightarrow e^{i t}} f(z)$ exist on $\partial U \backslash E$ and they are on $\partial \Omega$.

(c) The functions

$$
f^{*}\left(e^{i t-}\right)=\operatorname{ess} \lim _{s \uparrow t} f^{*}\left(e^{i s}\right) \quad \text { and } \quad f^{*}\left(e^{i t+}\right)=\operatorname{ess} \lim _{s \downarrow t} f^{*}\left(e^{i s}\right)
$$

exist on $\partial U$, belong to $\partial \Omega$ and are equal on $\partial U \backslash E$.

(d) The cluster set of $f$ at $e^{i t} \in E$ is the straight line segment joining $f^{*}\left(e^{i t-}\right)$ to $f^{*}\left(e^{i t+}\right)$.

The mapping $f$ is termed a Generalized Riemann Mapping (GRM) from $\mathbf{D}$ onto $\Omega$. It is immediate that the boundary function $f^{*}$ is continuous at every point in $\partial U \backslash E$ and has a jump discontinuity at every point in $E$. We will use the term jump to describe the behavior of $f^{*}$ at every point of $E$. Note that the cluster set of $f$ on an arc $J \subset \partial U$ induces a boundary positively directed arc in $\partial f(U)$; this arc is denoted, with abuse of notation, by $f^{*}(J)$.

The uniqueness of GRMs has been established recently for strictly starlike domains $\Omega$ with respect to some interior point [3]; that is, every ray through the point meets $\partial \Omega$ in one point only. Obviously, every convex domain is strictly starlike relative to any interior point.

\section{Main results}

The two approaches described above, the Poisson formula (2) on one hand and Theorem A on the other, call for a study of the interplay between the behavior of 
the boundary function $f^{*}$ of a harmonic mapping $f$ of form (1) on one hand and the dilatation function $a(z)$ on the other; globally, locally, and pointwise.

One immediately observes that for an arc $J \in \partial \Omega$ that is concave with respect to the interior of $\Omega$, for any admissible dilatation $a(z)$, the boundary function $f^{*}$ is continuous if merely because a jump would imply that the image domain of the corresponding GRM near $J$ will step outside $\Omega$ which is not possible. Hence a jump should be sought on a convex part of the boundary, if any. On the other hand, in Laugesen's proof of Theorem 5 [12, p. 47], if a jump occurs at a point $e^{i \theta_{0}} \in \mathbf{T}$, then it is noted that the angular limit of the dilatation $a(z)$ at $e^{i \theta_{0}}$ satisfies

$$
\lim _{z \angle e^{i \theta_{0}}} a(z)=\frac{\overline{d f^{*}\left(e^{i \theta_{0}}\right)}}{d f^{*}\left(e^{i \theta_{0}}\right)} .
$$

In particular this limit is of magnitude one. Analytic functions in the unit disk, bounded by one and having radial limits of magnitude one a.e. on $\mathbf{T}$ are inner functions. We focus attention primarily on inner function dilatations. A basic global result is

Theorem B. (Corollary 2, [12]) Let $\Phi(\theta)$ be the boundary function of a GRM that maps $\mathbf{D}$ onto a bounded convex set, and whose dilatation is $a(z)$. Then $\Phi^{\prime}(\theta)=0$ a.e. on $\partial D$ if, and only if, $a(z)$ is an inner function.

In [4] it is observed that certain Möbius transformations on an inner dilatation transform it into a Blaschke product, which results in another GRM of $\mathbf{D}$ onto a convex domain $\Omega$ with a Blaschke dilatation.

We shall examine the effect of the local property $\Phi^{\prime}\left(\theta_{0}\right)$ for some $\theta_{0} \in \partial D$ on Blaschke dilatations. In Theorem 1 and Theorem 2 we show that if $\left|\Phi^{\prime}\left(\theta_{0}\right)\right|$ is small enough, then the number of zeros of the complex dilatation $a(z)$ in any Stolz angle at $\theta_{0}$ is finite. Corollary 3 gives the effect of the above property on the boundary behavior of the analytic factor $h^{\prime}$ of $f$.

As to the pointwise behavior of the dilatation, let $B(z)$ be a (infinite) Blaschke product of the form (8). In such a case $B$ is uniquely determined by its zeros and a rotation. We shall examine the relationship of the zeros of $B$ and the jumps and continuity of $f^{*}$.

The immediate question which arises is this: To what extent one can force a jump of $f^{*}$ at a specific interval or a specific point?

In the special case where $B \equiv A$ is a finite Blaschke product, the corresponding GRM maps the disk onto a polygon and $f^{*}$ is a piecewise constant function with $n+2$ jumps. A mere rotation $e^{i \alpha} A$ of $A$ is enough to change a jump to continuity at a boundary point. If it is at all possible to specify the points on $\mathbf{T}$ where jumps occur in terms of $A$, this would be of interest. It turns out that a complete characterization of jumps is related to the analytic or co-analytic factors of the harmonic mapping $f(z)$ (Theorem 3).

We then assume that $B$ is an infinite Blaschke product. Given an interval $J \subset \mathbf{T}$, if the boundary function $f^{*}$ of the GRM $f$ associated with $B$ is constant on $J$, then for $B_{1}$, a Blaschke product with only one specific extra linear factor added to $B$, the boundary function $f_{1}^{*}$ of the associated GRM, can be made to have a jump in $J$ (Corollary 4 in [4]).

The pointwise behavior, however, is far more interesting. The answer has to do with the Frostman sum in the left hand side of (9). If the Frostman sum of $B$ is finite, 
one can generate a subproduct $B_{1}$ of $B$ such that the corresponding $f_{1}^{*}$ is continuous at $e^{i \theta_{0}}$ and by a mere rotation of $B_{1}, B_{2}=e^{i \alpha} B_{1}$, the corresponding boundary function $f_{2}^{*}$ of the GRM $f_{2}$ associated with $B_{2}$, admits a jump at $e^{i \theta_{0}}$ (Theorem 5 ). However, if the Frostman sum of $B$ at $e^{i \theta_{0}}$ is infinite, it was shown (Corollary 1 in [4]) that if the zeros of the Blaschke product are in one side of the radius to $e^{i \theta}$ then $f^{*}$ is continuous at $e^{i \theta_{0}}$. This result is extended to show that the same holds without restrictions on the placement of the zeros of the Blaschke product (Theorem 6). Note that this result refers to any GRM whose dilatation is a Blaschke product or any of its rotations. Theorem 4 gives another result ensuring the continuity of a GRM at a specific point.

\section{Differentiability at a boundary point}

A requisite for our results in this section is the following result due to Fatou [15, pp. 132-135].

Theorem C. (Fatou [15]) Let $u^{*}$ be an integrable real-valued function of $\mathbf{T}$ and let $u=P\left[u^{*}\right]$. Then:

(i) If $\left(d u^{*} / d \theta\right)\left(e^{i \theta_{0}}\right)$ exists and is finite, then the angular limit

$$
\lim _{z \angle e^{i \theta_{0}}} \frac{\partial u}{\partial \theta}(z)=\frac{d u^{*}}{d \theta}\left(e^{i \theta_{0}}\right)
$$

is uniform in any Stolz angle with vertex at $e^{i \theta_{0}}$.

(ii) If $\left(d u^{*} / d \theta\right)\left(e^{i \theta_{0}}\right)=+\infty$, then

$$
\lim _{r \rightarrow 1^{-}} \frac{\partial u}{\partial \theta}\left(r e^{i \theta}\right)=+\infty
$$

If, in addition, $u^{*}$ is monotone increasing in a neighborhood of $\theta_{0}$, then the angular limit

$$
\lim _{z \angle e^{i \theta_{0}}} \frac{\partial u}{\partial \theta}(z)=+\infty
$$

is uniform in any Stolz angle with vertex at $e^{i \theta_{0}}$.

(iii) If $\left(d u^{*} / d \theta\right)\left(e^{i \theta_{0}}\right)$ is continuous on an interval $[\alpha, \beta]$ and $\alpha<\alpha_{1}<\beta_{1}<\beta$, then $\partial u / \partial \theta \rightarrow d u^{*} / d \theta$ uniformly on $\left[\alpha_{1}, \beta_{1}\right]$ as $z \rightarrow e^{i \theta}$ from $\mathbf{D}$.

Evidently, in (ii) $-\infty$ may replace $+\infty$, and (i) and (iii) hold true for integrable complex-valued functions $f^{*}$ of $\mathbf{T}$.

The first result in this section is the following.

Lemma 1. Let $f^{*}$ be an integrable complex-valued function of $\mathbf{T}$ and let $f$ be the function defined by (2). Then $f$ is a harmonic mapping of $\mathbf{D}$ of the form $f=h+\bar{g}$, where $h$ and $g$ are analytic functions of $\mathbf{D}$, whose dilatation is denoted by a. If $\left(d f^{*} / d \theta\right)\left(e^{i \theta_{0}}\right)=0$ for some $\theta_{0}$ and the cluster set of $h^{\prime}$ at $e^{i \theta_{0}}$ does not contain zero, then the angular limit of $|a|$ at $e^{i \theta_{0}}$ is 1 and a can have only a finite number of zeros in any Stolz angle with vertex $e^{i \theta_{0}}$.

Proof. Let $f$ be as in (2) and have dilatation $a(z)$. Then

$$
\frac{\partial f}{\partial \theta}(z)=i\left(z h^{\prime}(z)-\overline{z g^{\prime}(z)}\right) .
$$


By invoking Theorem $\mathrm{C}$ at $\theta_{0}$ provided that the cluster set of $h^{\prime}$ at $e^{i \theta_{0}}$ does not contain zero, we conclude that

$$
0=-i \lim _{z \angle e^{i \theta_{0}}} \frac{\partial f}{\partial \theta}(z)=\lim _{z \angle e^{i \theta_{0}}} z h^{\prime}(z)\left(1-\frac{\overline{z h^{\prime}(z)}}{z h^{\prime}(z)} \overline{a(z)}\right)=\lim _{z \angle e^{i \theta_{0}}}\left(1-\overline{z h^{\prime}(z)} \overline{z h^{\prime}(z)} \overline{a(z)}\right),
$$

where the limits involved are angular limits. This implies at once that the angular limit of $|a|$ at $e^{i \theta_{0}}$ is 1 .

The assumption that the cluster set of $h^{\prime}$ at $e^{i \theta_{0}}$ does not contain zero yields at once that $h^{\prime}$ has only a finite number of zeros in any Stolz angle $S_{\theta_{0}}$ at $e^{i \theta_{0}}$. Suppose that $g^{\prime}$ has infinitely many zeros in $S_{\theta_{0}}$. Then infinitely many of these zeros are not zeros for $h^{\prime}$; hence are zeros of $a$ which contradicts the conclusion of the previous paragraph. Thus $g^{\prime}$, like $h^{\prime}$, has a finite number of zeros in $S_{\theta_{0}}$. But every zero of $a$ is a zero of $g^{\prime}$. Therefore $a$ has only a finite number of zeros in $S_{\theta_{0}}$ and the proof is complete.

We shall combine Lemma 1 with an extension of a theorem of Heinz [9] due to Kalaj [11, Theorem 2.5].

Theorem D. Let $f$ be a univalent harmonic mapping of $\mathbf{D}$ onto a bounded convex domain $\Omega$ containing the origin and let $f(0)=0$. Then

$$
\left|f_{z}\right|^{2}+\left|f_{\bar{z}}\right|^{2} \geq \frac{\operatorname{dist}(0, \partial \Omega)^{2}}{16} .
$$

By combining Theorem D with Lemma 1 we obtain the following interesting consequence.

Theorem 1. Let $f$ be the GRM from the unit disc $\mathbf{D}$ onto a bounded convex set with boundary function $f^{*}$ and dilatation $a$. If $\left(d f^{*} / d \theta\right)\left(e^{i \theta_{0}}\right)=0$ for some $\theta_{0} \in \mathbf{R}$, then the angular limit of $|a|$ at $e^{i \theta_{0}}$ is 1 ; in particular, a has at most a finite number of zeros in any Stolz angle with vertex $e^{i \theta_{0}}$.

Proof. By Theorem D it follows that $h^{\prime}$ is bounded away from 0 , so by Lemma 1 the result follows.

As a special case of Theorem 1 we have

Corollary 1. Let $f$ of form (1) be the GRM from the unit disc $\mathbf{D}$ onto a bounded convex set with boundary function $f^{*}$ and dilatation $a$. If $\left(d f^{*} / d \theta\right)\left(e^{i \theta_{0}}\right)=0$ for some $\theta_{0} \in \mathbf{R}$ and the angular $\operatorname{limit}_{\lim _{z} e^{i \theta_{0}}} \arg a(z)=\alpha$, then the angular limits

$$
\lim _{z \angle e^{i \theta_{0}}} \arg h^{\prime}(z)=-\theta_{0}-\frac{1}{2} \alpha \quad(\bmod \pi)
$$

and

$$
\lim _{z \angle e^{i \theta_{0}}} \arg g^{\prime}(z)=-\theta_{0}+\frac{1}{2} \alpha \quad(\bmod \pi)
$$

hold.

Proof. As in the proof of Lemma 1, the angular limit

$$
\lim _{z \angle e^{i \theta_{0}}}\left(1-\overline{z h^{\prime}(z)} \overline{z h^{\prime}(z)} \overline{a(z)}\right)=0
$$


holds. Consequently, the angular limit

$$
\lim _{z \angle e^{i \theta_{0}}} \arg \left\{\frac{\overline{z h^{\prime}(z)}}{z h^{\prime}(z)} \overline{a(z)}\right\}=0
$$

holds. This gives the desired limits.

The next result reveals some properties in the case where $\left(d f^{*} / d \theta\right)\left(e^{i \theta_{0}}\right)$ is nonzero and finite.

Corollary 2. Let $f^{*}$ be an integrable complex-valued function of $\mathbf{T}$ and let $f$ of form (1) be the harmonic mapping of $\mathbf{D}$ defined by (2) and have dilatation $a$. Suppose that $\left(d f^{*} / d \theta\right)\left(e^{i \theta_{0}}\right)=\alpha \neq 0, \infty$ for some $\theta_{0}$ and the angular limit $\lim _{z<e^{i \theta_{0}}} h^{\prime}(z)=\beta$ exists. Then $\beta \neq 0$ and the angular limit $\lim _{z<e^{i \theta_{0}}} a(z)$ exists.

Proof. By Theorem C, the angular limit

$$
\lim _{z \angle e^{i \theta_{0}}} \frac{\partial f}{\partial \theta}(z)=\frac{d f^{*}}{d \theta}\left(e^{i \theta_{0}}\right)=\alpha .
$$

But

$$
-i \frac{\partial f}{\partial \theta}(z)=z h^{\prime}(z)-\overline{z g^{\prime}(z)}=z h^{\prime}(z) q(z)
$$

where

$$
q(z)=\left[1-\frac{\overline{z h^{\prime}(z)}}{z h^{\prime}(z)} \overline{a(z)}\right] .
$$

Since $q$ is bounded in $\mathbf{D}$, by taking the angular limits of both sides of (4) at $e^{i \theta_{0}}$ we obtain $\beta \neq 0$. Moreover, we infer that the angular limit of $q$ at $e^{i \theta_{0}}$ exists; and consequently that of $a$.

In the special case of GRM's onto convex domains we have the following result:

Theorem 2. Let $f$ be the GRM from the unit disc $\mathbf{D}$ onto a bounded convex set with boundary function $f^{*}$ and dilatation $a$. If $\left(d f^{*} / d \theta\right)\left(e^{i \theta_{0}}\right)=\alpha \neq 0$ for some $\theta_{0} \in \mathbf{R}$, and sufficiently small constant $|\alpha|$, then the $a$ has at most a finite number of zeros in any Stolz angle with vertex $e^{i \theta_{0}}$.

Proof. From (7) and Theorem $\mathrm{C}$ we conclude that

$$
\lim _{z \angle e^{i \theta_{0}}}\left|h^{\prime}(z)-\overline{g^{\prime}(z)}\right|=|\alpha|,
$$

and in particular

$$
\varlimsup_{z \angle e^{i \theta_{0}}}\left(\left|h^{\prime}(z)\right|-\left|g^{\prime}(z)\right|\right)|\leq| \alpha \mid
$$

so that

$$
\varlimsup_{z \angle e^{i \theta_{0}}}\left|g^{\prime}(z)\right|\left|\geq \varlimsup_{z \angle e^{i \theta_{0}}}\right| h^{\prime}(z)|-| \alpha \mid .
$$

As in the proof of Theorem 1 , by Theorem $\mathrm{D}$ there exists a positive constant $c$ such that $\left|h^{\prime}(z)\right| \geq c$. Thus, for any $\alpha$ with $|\alpha|<c$, we must have $\underline{\lim }_{z<e^{i \theta_{0}}}\left|g^{\prime}(z)\right|>0$ so that $a$ has finitely many zeros in any Stolz angle at $e^{i \theta_{0}}$.

It would be interesting to know whether for other values of $d f^{*} / d \theta$ the dilatation function has finitely many zeros in Stolz angles. 


\section{Continuity at a boundary point}

We start with a characterization of the continuity and the jump of a GRM at a boundary point. We have the following result:

Lemma 2. Let $f^{*}$ be a complex-valued function of bounded variation on $\mathbf{T}$ and let $f$ of form (1) be the harmonic mapping of $\mathbf{D}$ defined by (2) and have dilatation a. Then $\lim _{r \rightarrow 1^{-}}(1-r) h^{\prime}\left(r e^{i \theta_{0}}\right)=c$ exists if and only if $\lim _{r \rightarrow 1^{-}}(1-r) g^{\prime}\left(r e^{i \theta_{0}}\right)=d$ exists; in this case $c$ and $d$ are finite and $c e^{i \theta_{0}}=\overline{d e^{i \theta_{0}}}$. In particular, either limit is zero if and only if $f^{*}$ is continuous at $e^{i \theta_{0}}$.

Remark 1. Note that this result asserts the equivalence of the continuity of $f^{*}$ and the radial growth of $h^{\prime}$, or equivalently $g^{\prime}$, and not the dilatation $a$, at a boundary point. A jump of size $b>0$ occurs at $e^{i \theta_{0}}$, if and only if

$$
\lim _{r \rightarrow 1^{-}}(1-r)\left|h^{\prime}\left(r e^{i \theta_{0}}\right)\right|=\lim _{r \rightarrow 1^{-}}(1-r)\left|g^{\prime}\left(r e^{i \theta_{0}}\right)\right|=b .
$$

Proof. Differentiating (2) with respect to $z$ and $\bar{z}$ and using integration by parts we obtain

$$
h^{\prime}(z)=\frac{1}{2 \pi} \int_{0}^{2 \pi} \frac{d f^{*}}{e^{i \phi}-z} \quad \text { and } \quad g^{\prime}(z)=\frac{1}{2 \pi} \int_{0}^{2 \pi} \frac{\overline{d f^{*}}}{e^{i \phi}-z} .
$$

Suppose that $\lim _{r \rightarrow 1^{-}}(1-r) h^{\prime}\left(r e^{i \theta_{0}}\right)=c$ exists, then, by the bounded convergence theorem,

$$
e^{i \theta_{0}} c=e^{i \theta_{0}} \lim _{r \rightarrow 1^{-}}(1-r) h^{\prime}\left(r e^{i \theta_{0}}\right)=\lim _{r \rightarrow 1^{-}} \frac{1}{2 \pi} \int_{0}^{2 \pi} \frac{1-r}{e^{i\left(\phi-\theta_{0}\right)}-r} d f^{*}=d f^{*}\left(e^{i \theta_{0}}\right) .
$$

But $f^{*}$ is a function of bounded variation over $[0,2 \pi]$; hence $d f^{*}\left(e^{i \theta_{0}}\right)$ is finite; so likewise is $c$. In view of this, and using essentially the same argument, we conclude that

$$
e^{i \theta_{0}} d=e^{i \theta_{0}} \lim _{r \rightarrow 1^{-}}(1-r) g^{\prime}\left(r e^{i \theta_{0}}\right)=\overline{d f^{*}\left(e^{i \theta_{0}}\right)} .
$$

Suppose now that $\lim _{r \rightarrow 1^{-}}(1-r) g^{\prime}\left(r e^{i \theta_{0}}\right)=d$ exists, then a similar argument as above yields that $\lim _{r \rightarrow 1^{-}}(1-r) h^{\prime}\left(r e^{i \theta_{0}}\right)=c$ exists and that $c e^{i \theta_{0}}=\overline{d e^{i \theta_{0}}}$. This concludes the first claim of the theorem. The second claim then follows at once and the proof is complete.

An immediate consequence of this theorem is the following interesting result:

Theorem 3. Let $f$ of form (1) be a GRM from $\mathbf{D}$ onto a bounded Jordan domain with a rectifiable boundary, and let $a$ and $f^{*}$ be the dilatation and boundary function of $f$ respectively. Then $\lim _{r \rightarrow 1^{-}}(1-r) h^{\prime}\left(r e^{i \theta_{0}}\right)=c$ and $\lim _{r \rightarrow 1^{-}}(1-r) g^{\prime}\left(r e^{i \theta_{0}}\right)=d$ exist, are finite, and satisfy $c e^{i \theta_{0}}=\overline{d e^{i \theta_{0}}}$; moreover, either limit is zero if and only if $f^{*}$ is continuous at $e^{i \theta_{0}}$.

Proof. In the characterization of $f^{*}$ given in Theorem A, the set $E$ of jumps must be empty and thus $f^{*}$ is continuous on $\mathbf{T}$.

We conclude at once that a GRM $f^{*}$ is continuous at $e^{i \theta_{0}}$ if $f$ satisfies the additional condition that either one of the cluster sets of $h^{\prime}$, or $g^{\prime}$, at $e^{i \theta_{0}}$ is away from infinity. Another consequence of Lemma 2 is the following result:

Corollary 3. Under the assumptions of Lemma 2, suppose that there exists a sequence $\left\{z_{n}\right\}$ of complex numbers in $\mathbf{D}$ that satisfies the following properties: 
(a) $\lim _{n \rightarrow \infty} z_{n}=e^{i \theta_{0}}$ for some $\theta_{0} \in \mathbf{R}$;

(b) $\lim _{n \rightarrow \infty}\left|z_{n}-z_{n-1}\right| /\left(1-\left|z_{n}\right|\right)=0$;

(c) $\lim _{n \rightarrow \infty} g^{\prime}\left(z_{n}\right)=\alpha$ exists and is finite.

Then $f^{*}$ is continuous at $e^{i \theta_{0}}$.

Proof. Conditions (a) and (b) imply that $\lim _{n \rightarrow \infty} \rho\left(z_{n}, z_{n-1}\right)=0$, where $\rho\left(z_{n}\right.$, $\left.z_{n-1}\right)$ denotes the hyperbolic distance between $z_{n}$ and $z_{n-1}$. Now, $g^{\prime}(z)$ is a normal function since

$$
(1-|z|)\left|g^{\prime}(z)\right| \leq \frac{1}{2 \pi} \int_{0}^{2 \pi} \frac{1-|z|}{\left|e^{i \phi}-z\right|}\left|d f^{*}\left(e^{i \phi}\right)\right| \leq \frac{1}{2 \pi} \int_{0}^{2 \pi}\left|d f^{*}\left(e^{i \phi}\right)\right| \leq L<\infty .
$$

where $L$ is the length of $\partial f(\mathbf{D})$. By a result of Bagemihl-Seidel [1], $\lim _{r \rightarrow 1^{-}} g^{\prime}\left(r e^{i \theta_{0}}\right)=$ $\alpha$; hence $\lim _{r \rightarrow 1^{-}}(1-r) g^{\prime}\left(r e^{i \theta_{0}}\right)=0$. Therefore, $f^{*}$ is continuous at $e^{i \theta_{0}}$ by Theorem 3 and the proof is complete.

A special case of the above corollary is when the values $z_{n}$ are zeros of $g^{\prime}$ which are the same as the zeros of $a$.

Theorem 4. Let $f$ of form (1) be a GRM from $\mathbf{D}$ onto a Jordan domain having a rectifiable boundary, and let $a$ and $f^{*}$ are the dilatation and boundary values of $f$ respectively. If $\left\{z_{n}\right\}$ is a sequence satisfying (a) and (b) of Corollary 3 , and $a\left(z_{n}\right)=0$, $n=1,2,3, \cdots$, then $f^{*}$ is continuous at $e_{0}^{i \theta}$.

In the next result, we illustrate the significance of Theorem 3 by showing the surprising fact that the set of zeros of the dilatation of the GRM $f$ from $\mathbf{D}$ onto $\mathbf{D}$ need not determine the behavior of the boundary function $f^{*}$. We shall take $B(z)$ to be a Blaschke product

$$
B(z)=e^{i \alpha} z^{m} \prod_{n=m+1}^{\infty} \frac{\left|\zeta_{n}\right|}{\zeta_{n}} \frac{\zeta_{n}-z}{1-\bar{\zeta}_{n} z},
$$

where $m$ is a nonnegative integer and $\left\{\zeta_{n}\right\}$ is an infinite sequence of nonzero complex numbers satisfying $\sum\left(1-\left|\zeta_{n}\right|\right)<\infty$.

Theorem 5. Suppose the following:

(a) The numbers $\zeta_{n} \in \mathbf{D}, n=1,2, \cdots$, are such that $\Re\left(e^{-i \theta_{0}} \zeta_{n}\right)>0$ for some $\theta_{0} \in \mathbf{R}$, and

$$
\sum_{n=1}^{\infty} \frac{1-\left|\zeta_{n}\right|}{\left|e^{i \theta_{0}}-\zeta_{n}\right|}<\infty
$$

(b) The Blaschke subproducts $B_{j}^{N}(z)$ are defined by

$$
B_{j}^{N}(z)=e^{i \alpha_{j}} \prod_{n=N}^{\infty} \frac{\left|\zeta_{n}\right|}{\zeta_{n}} \frac{\zeta_{n}-z}{1-\bar{\zeta}_{n} z}, j=1,2 .
$$

(c) The $f_{j}$ are GRMs from $\mathbf{D}$ onto $\mathbf{D}$, with $f_{j}(0)=0$, and whose dilatation is some $B_{j}^{N}$ for some chosen $N$.

Then for sufficiently large $N$, there exist values $\alpha_{j}$ such that the boundary function $f_{j}^{*}$ of $f_{j}$ has a jump discontinuity at $e^{i \theta_{0}}$ for some value of $\alpha_{j}$ and is continuous at $e^{i \theta_{0}}$ for another. 
The condition $\Re\left(e^{-i \theta_{0}} \zeta_{n}\right)>0$ may be replaced by the condition $\Re\left(e^{-i \theta_{0}} \zeta_{n}\right)<0$; in this case the desired GRM has form $\overline{f(\bar{z})}$, where $f$ is the desired GRM whose Blaschke product is $\overline{B(\bar{z})}$, with zeros $\overline{\zeta_{n}}$ satisfying $\Re\left(e^{i \theta_{0}} \overline{\zeta_{n}}\right)>0$ and $\overline{\zeta_{n}} \rightarrow e^{-i \theta_{0}}$. In view of this, either condition becomes unnecessary for the validity of Theorem 5 provided that the Blaschke products $B_{j}^{N}$ are chosen as subproducts of $B$ with a common set of zeros, say $\left\{\zeta_{n_{k}}\right\}$; namely a set that satisfies for all $k$ either $\Re\left(e^{-i \theta_{0}} \zeta_{n_{k}}\right)>0$ or $\Re\left(e^{-i \theta_{0}} \zeta_{n_{k}}\right)<0$.

We shall use the notation $\arg \sqrt{a(z)}$ and $\Delta_{J} \arg \sqrt{a(z)}$ to denote a single-valued branch of $\arg \sqrt{a}$ and its net variation on a subarc $J$ of the unit circle.

The proof of Theorem 5 requires some known results.

Lemma 3. (Bshouty, Lyzzaik, Weitsman [4]) Let $a(z)$ be a Blaschke product whose zeros $\left\{\zeta_{n}\right\}$ accumulate at 1 , and let $J=\left\{e^{i t}:-\gamma<t<0\right\}$, where $0<\gamma<\pi$.

(a) If $\Im \zeta_{n}>0$ for all $n$, then

$$
\Delta_{J} \arg \sqrt{a(z)}=\frac{1}{2} \sum_{n=1}^{\infty} \Delta_{J} \arg \left\{\frac{z-\zeta_{n}}{1-\overline{\zeta_{n}} z}\right\} \sim \sum_{n=1}^{\infty} \frac{1-\left|\zeta_{n}\right|}{\left|1-\zeta_{n}\right|} ;
$$

this means that the expressions on both of sides of $\sim$ converge or diverge simultaneously.

(b) If infinitely many $\zeta_{n}$ satisfy $\Im \zeta_{n} \leq 0$, then $\Delta_{J} \arg \sqrt{a(z)}=\infty$.

Under the assumptions of Lemma 3 the Blaschke product $a$ extends analytically across $\partial \mathbf{D} \backslash\{1\}$, with $|a|=1$ there, but not across 1 .

Lemma 4. Let $f$ be a GRM from $\mathbf{D}$ onto a bounded convex domain $\Omega$ whose dilatation a admits an analytic extension across an open interval $J=\left\{e^{i t}: \gamma<t<\right.$ $\delta\}, \gamma<\delta<\gamma+2 \pi$, such that $|a| \equiv 1$ on $J$. Then the following hold:

(a) $f^{*}$ has a jump at $e^{i \theta} \in J$ if and only if $\arg \left\{\sqrt{a\left(e^{i \theta}\right)} d f^{*}\left(e^{i \theta}\right)\right\}=0 \bmod \pi$.

(b) $\Delta_{J} \arg \sqrt{a(z)}=\infty$ if and only if $f^{*}$ has infinitely many jumps in $J$.

(c) If $f^{*}$ has no jumps in $J$, then $f^{*}$ is identically constant on $J$.

Note that Lemma 4 (a) and (c) follow from Theorem 2.2 and Corollary 2.8 respectively of [2], and Lemma 4 (b) is itself Corollary 2(i) of [4].

Proof of Theorem 5. First, we assert that the theorem can be reduced to the case where $\theta_{0}=0$ and each $f_{j}^{*}\left(e^{i 0+}\right)=1$; note that $\left|f_{j}^{*}\left(e^{i \theta+}\right)\right|=\left|f_{j}^{*}\left(e^{i \theta-}\right)\right|=1$ for every $\theta \in \mathbf{R}$ by Theorem 1 . For if $f_{j}(z)$ is the GRM from $\mathbf{D}$ onto $\mathbf{D}$ with $f_{j}(0)=0$, $f_{j}^{*}\left(e^{i 0+}\right)=1$, and dilatation $B_{j}^{N}\left(e^{2 i \theta_{0}} z\right)$, then it can be easily verified that every $g_{j}(z)=e^{i \gamma} f_{j}\left(e^{i \theta_{0}} z\right)$ is the GRM from $\mathbf{D}$ onto $\mathbf{D}$ with $g_{j}(0)=0, g_{j}^{*}\left(e^{i \theta_{0}+}\right)=e^{i \gamma}$, and dilatation $B_{j}^{N}(z)$.

Thus we assume henceforth that $\theta_{0}=0$ and each $f_{j}\left(e^{i 0+}\right)=1$. Let $\partial \mathbf{D}_{-}=$ $\partial \mathbf{D} \cap\{z: \Im z<0\}$.

We divide the proof into two parts:

A. Here we find the value $\alpha_{1}$ such that for sufficiently large $N$ the boundary function $f_{1}^{*}$ of the GRM $f_{1}$ has a jump at 1 .

Since each $\Im \zeta_{n}>0$, Lemma $3(\mathrm{a})$ and (9) yield

$$
\Delta_{\partial \mathbf{D}_{-}} \arg \sqrt{B_{1}^{N}(z)}=\frac{1}{2} \sum_{n=N}^{\infty} \Delta_{\partial \mathbf{D}_{-}} \arg \left\{\frac{z-\zeta_{n}}{1-\overline{\zeta_{n}} z}\right\} \sim \sum_{n=N}^{\infty} \frac{1-\left|\zeta_{n}\right|}{\left|1-\zeta_{n}\right|}<\infty .
$$


Hence, for a fixed sufficiently large $N$ we have

$$
\Delta_{\partial \mathbf{D}_{-}} \arg \sqrt{B_{1}^{N}(z)}<\frac{\pi}{2}
$$

Now we drop reference to $N$ and choose $\alpha_{1}$ so that $0<\arg \sqrt{B_{1}(z)}<\pi / 2$ for all $z \in \partial \mathbf{D}_{\text {-. }}$. Define $f_{1}$ as stated in the theorem. By virtue of Lemma 3 (b) and Lemma 4 (b), we conclude that $f_{1}^{*}$ attains countably many jumps at points $e^{i \theta_{n}}, n=1,2, \cdots$, satisfying

$$
2 \pi>\theta_{1}>\cdots>\theta_{n}>\cdots \text { and } \lim _{n \rightarrow \infty} \theta_{n}=0 .
$$

It is immediate from Lemma 4 (c) that $f_{1}^{*}$ is identically a unimodular constant $\omega$ for all $e^{i \theta}, \theta_{1}<\theta<2 \pi$.

To show that $f_{1}^{*}$ has a jump discontinuity at 1 , it is enough to establish that $\omega \neq 1$. Suppose to the contrary that $\omega=1$. Then $\pi<\theta_{1}<2 \pi$ since, by the mean-value property applied to $\Re f_{1}$, we have

$$
\begin{aligned}
0 & =2 \pi f_{1}(0)=2 \pi \Re f_{1}(0)=\int_{0}^{\theta_{1}} \Re f_{1}^{*}\left(e^{i \theta}\right) d \theta+\int_{\theta_{1}}^{2 \pi} \Re f_{1}^{*}\left(e^{i \theta}\right) d \theta \\
> & \int_{0}^{\theta_{1}}-d \theta+\int_{\theta_{1}}^{2 \pi} d \theta=2\left(\pi-\theta_{1}\right) .
\end{aligned}
$$

Thus $\arg \sqrt{B_{1}^{N}\left(e^{i \theta_{1}}\right)}<\pi / 2$. Moreover, since the origin belongs to $f_{1}(\mathbf{D}), f_{1}^{*}\left(e^{i \theta_{1}-}\right) \in$ $\partial \mathbf{D}_{-}$; hence $0<\arg d f_{1}^{*}\left(e^{i \theta_{1}}\right)<\pi / 2$. It follows that

$$
0<\arg \left\{\sqrt{B_{1}^{N}\left(e^{i \theta_{1}}\right)} d f_{1}^{*}\left(e^{i \theta_{1}}\right)\right\}<\pi / 2+\pi / 2=\pi
$$

which, by Lemma 4 (a), contradicts the assumption that $e^{i \theta_{1}}$ is a discontinuity point for $f_{1}^{*}$. Therefore, $\omega \neq 1$ and $f_{1}^{*}$ is discontinuous at 1 as desired.

B. Here we find the value $\alpha_{2}$ such that for sufficiently large $N$ the boundary function $f_{2}^{*}$ of the GRM $f_{2}$ is continuous at 1 .

Let $B_{1}^{N}$ be as found in part $\mathrm{A}$; so $0<\arg \sqrt{B_{1}^{N}(z)}<\pi / 2$ for all $z \in \partial \mathbf{D}_{-}$. By invoking (9), we infer that $B_{1}^{N *}(1)=\lim _{r \rightarrow 1^{-}} B_{1}^{N}(r)$ exists and $\left|B^{N *}(1)\right|=1$; see $[4$, Theorem D]. Let $\alpha_{2}=-\arg B_{1}^{N *}(1)$ and let $B_{2}^{N}(z)=e^{i \alpha_{2}} B_{1}^{N}(z)$; hence $B_{2}^{N *}(1)=1$ and

$$
\Delta_{\partial \mathbf{D}_{-}} \arg \sqrt{B_{2}^{N}(z)}<\pi / 2 .
$$

Define $f_{2}$ as stated in the theorem. Then we conclude, as above in part A, that $f_{2}^{*}$ attains countably many jumps at points $e^{i \theta_{n}}, n=1,2, \cdots$, satisfying (10) such that $f_{2}^{*}$ is identically a unimodular constant $\omega$ for all $e^{i \theta}, \theta_{1}<\theta<2 \pi$.

To show that $f_{2}^{*}$ is continuous at 1 , it is enough to establish that $\omega=1$. Suppose that $\Im \omega>0$; then $f_{2}^{*}\left(e^{i 0-}\right)=\omega$. But $f_{2}^{*}\left(e^{i 0+}\right)=1$. Hence, the directed straight line segment $[\omega, 1]$ is a boundary arc of $f(\mathbf{D})$ that has the origin on its right-hand side. But the origin lies in $f(\mathbf{D})$ and $f_{2}$ is sense-preserving in $\mathbf{D}$; hence the origin lies on the left-hand side of $[\omega, 1]$ and we have a contradiction.

It follows that $\Im \omega \leq 0$. Suppose now that $\Im \omega<0$. Since $f_{2}^{*}\left(e^{i 0-}\right)=\omega \neq 1=$ $f_{2}^{*}\left(e^{i 0+}\right), f_{2}^{*}$ admits a jump at 1 with the cluster set of $f_{2}$ at 1 is the straight line segment $[\omega, 1]$. Thus, $0<\arg d f_{2}^{*}(1)<\pi / 2$. Assuming that $f_{2}^{*}\left(e^{i t}\right), 0 \leq t \leq 2 \pi$, is 
right-continuous on $[0,2 \pi]$ and that $f_{2}=h_{2}+\overline{g_{2}}$, then, by [12, p. 47], we have

$$
B_{2}^{N}(r)=\frac{(1-r) g_{2}^{\prime}(r)}{(1-r) h_{2}^{\prime}(r)}=\int_{-\pi}^{\pi} \frac{(1-r) \overline{d f_{2}^{*}\left(e^{i t}\right)}}{e^{i t}-r} / \int_{-\pi}^{\pi} \frac{(1-r) d f_{2}^{*}\left(e^{i t}\right)}{e^{i t}-r} .
$$

But $\int_{-\pi}^{\pi}\left|d f_{2}^{*}\left(e^{i t}\right)\right|<2 \pi$. Hence, by the Lebesgue dominated convergence theorem,

$$
1=B_{2}^{N *}(1)=\lim _{r \rightarrow 1} B_{2}^{N *}(r)=\frac{\overline{d f_{2}^{*}(1)}}{d f_{2}^{*}(1)},
$$

which yields $\arg d f_{2}^{*}(1)=0$ and we have a contradiction. Therefore, $f_{2}^{*}\left(e^{i 0-}\right)=\omega=$ $1=f_{2}^{*}\left(e^{i 0+}\right)$ and $f_{2}^{*}$ is continuous at 1 . This completes the proof of Theorem 5 .

Finally, we have the following theorem that completes our results Theorem 1 (b) and Corollary 1 in [3].

Lemma 5. (Protas [14]) Let $B$ be an infinite Blaschke product with zeros $\left\{\zeta_{n}\right\}$, and let $\zeta \in \partial \mathbf{D}, \gamma \geq 1$, and $m>0$. Then (9) holds if and only if

$$
\int_{\Gamma_{\zeta, \gamma, m}} \frac{1-|B(z)|^{2}}{1-|z|^{2}}|d z|<\infty,
$$

where $\Gamma_{\zeta, \gamma, m}=\Gamma$ is the "curve" in $\mathbf{D}$ defined by $\Gamma(\theta)=(1-m|\theta| \gamma) e^{i \theta}$ for $0<|\theta|<$ $\min \left\{\pi, m^{-1 / \gamma}\right\}$.

Theorem 6. Let $f$ of form (1) be a GRM from $\mathbf{D}$ onto a bounded convex domain $\Omega$, whose dilatation is a Blaschke product a with zeros $\zeta_{n}, n=1,2, \cdots$, and whose boundary function is $f^{*}$. If

$$
\sum_{n=1}^{\infty} \frac{1-\left|\zeta_{n}\right|}{\left|e^{i \theta_{0}}-\zeta_{n}\right|}=\infty
$$

then $f^{*}$ is continuous at $\theta_{0}$.

Proof. Without loss of generality we shall assume that $\theta_{0}=0$. Let us assume to the contrary that $f$ admits a jump at 1 . Then

$$
\lim _{z \angle 1}(1-|z|)\left|h^{\prime}(z)\right|=\lim _{z \angle 1}(1-|z|)\left|g^{\prime}(z)\right|=c_{1}>0,
$$

for some $c_{1}$. By Lemma $5,(11)$ is equivalent to

$$
\infty=\int_{\Gamma_{m}} \frac{1-|a(z)|^{2}}{1-|z|^{2}}|d z|,
$$

where $\Gamma_{m}(\theta)=(1-m|\theta|) e^{i \theta}, 0<|\theta| \leq \min \{\pi, 1 / m\}$, for every $m>0$. Let

$$
\Gamma_{m}^{+}(\theta)=(1-m \theta) e^{i \theta}, \quad 0<\theta \leq \min \{\pi, 1 / m\}
$$

and

$$
\Gamma_{m}^{-}(\theta)=(1+m \theta) e^{i \theta}, \quad-\min \{\pi, 1 / m\} \leq \theta<0 .
$$

We may observe the following properties of the "curves" $\Gamma_{m}$ :

(a) Each $\Gamma_{m}^{+}$is a closed-open Jordan arc that starts from the origin if $m \geq 1 / \pi$ and from a point in $(-1,0)$ if $0<m<1 / \pi$, terminates at 1 , and lies otherwise in the upper-half unit disc.

(b) $\Gamma_{m}^{+}$and $\Gamma_{m}^{-}$are symmetric about the real axis for every $m$.

(c) The arcs $\Gamma_{m}^{+}\left(\Gamma_{m}^{-}\right)$are mutually disjoint except when $m \geq 1 / \pi$ in which case they share the origin as an initial point. 
(d) If $R_{m}$ is the Jordan region bounded by $\Gamma_{m}$, then $R_{m}$ properly contains $R_{m^{\prime}}$ whenever $m^{\prime}>m$ and $\bigcap_{m} R_{m}$ is the open unit interval.

In view of Theorem $\mathrm{D}, h^{\prime}(z)$ is bounded away from zero in $\mathbf{D}$. This with (12), yields that $\left|h^{\prime}(z)\right|(1-|z|)$ is bounded away from zero. For the rest of the proof let $m \leq 1 / 2 \pi$ and $|\theta| \leq \pi$. We have

$$
\left|d \Gamma_{m}(\theta)\right|=|m+i(1-m|\theta|)| d \theta \leq(m+1) d \theta<2 d \theta, \quad|\theta| \leq \pi .
$$

Hence, by employing (13), we obtain

$$
\begin{aligned}
\infty & =\int_{\Gamma_{m}} \frac{\left(\left|h^{\prime}(z)\right|^{2}-\left|g^{\prime}(z)\right|^{2}\right)(1-|z|)}{\left|h^{\prime}(z)\right|^{2}(1-|z|)^{2}(1+|z|)}|d z| \leq c_{2} \int_{\Gamma_{m}}\left(\left|h^{\prime}(z)\right|^{2}-\left|g^{\prime}(z)\right|^{2}\right) m|\theta||d z| \\
& <c_{2} \int_{-\pi}^{\pi}\left(\left|h^{\prime}(z)\right|^{2}-\left|g^{\prime}(z)\right|^{2}\right)|\theta| d \theta
\end{aligned}
$$

for some real constant $c_{2}>0$.

By virtue of the properties (a), $\cdots,(d)$ of $\Gamma_{m}$, we infer that the mapping $\Gamma(m, \theta):(m, \theta) \rightarrow(r, \theta)$ defined by $\Gamma(m, \theta)=\Gamma_{m}(\theta)$ for $0<|\theta| \leq \pi$ is a diffeomorphism from the open rectangle $R=(-\pi, \pi) \times(0,1 / 2 \pi)$ minus the line segment $\{(m, 0): 0<m<1 / 2 \pi\}$ onto $Q=\mathbf{D} \backslash R_{1 / 2 \pi}$ minus the open interval $(-1,-1 / 2)$. But the Jacobian of $\Gamma(m, \theta)$ is $\partial(r, \theta) / \partial(m, \theta)=|\theta|$ and $1-m|\theta|>1 / 2$ in $Q$. Hence, the area $A$ of $f(\mathbf{D})$ satisfies

$$
\begin{aligned}
A & =\iint_{\mathbf{D}}\left(\left|h^{\prime}(z)\right|^{2}-\left|g^{\prime}(z)\right|^{2}\right) r d r d \theta>\iint_{Q}\left(\left|h^{\prime}(z)\right|^{2}-\left|g^{\prime}(z)\right|^{2}\right) r d r d \theta \\
& =\iint_{R}\left(\left|h^{\prime}(z)\right|^{2}-\left|g^{\prime}(z)\right|^{2}\right)(1-m|\theta|) \frac{\partial(r, \theta)}{\partial(m, \theta)} d m d \theta \\
& >\frac{1}{2} \int_{-\pi}^{\pi} \int_{0}^{1 / 2 \pi}\left(\left|h^{\prime}(z)\right|^{2}-\left|g^{\prime}(z)\right|^{2}\right)|\theta| d \theta d m=\infty .
\end{aligned}
$$

This yields a contradiction since $A$ is finite because $f(\mathbf{D}) \subset \Omega$. This completes the proof.

Remark 2. Let $\phi=z^{n} B(z) s(z)$ be an inner function where $B$ is a Blaschke product and $s$ is a singular inner function associated with the measure $\sigma$. Using Theorem 2 of Protas [14] and the preceding proof one can show the following:

Let $\phi$ be an inner function and let $f$ be the GRM from $\mathbf{D}$ onto a bounded convex domain associated with $\phi$. If

$$
\sum_{n=1}^{\infty} \frac{1-\left|\zeta_{n}\right|}{\left|e^{i \theta_{0}}-\zeta_{n}\right|}+\int_{0}^{2 \pi}\left|1-e^{i\left(t-\theta_{0}\right)}\right| d \sigma(t)=\infty
$$

then the boundary function $f^{*}$ is continuous at $e^{i \theta_{0}}$.

\section{References}

[1] Bageminl, F., and W. Seidel: Sequential and continuous limits of meromorphic functions. - Ann. Acad. Sci. Fenn. Ser. A I Math. 280, 1960, 1-17.

[2] Bshouty, D., and W. Hengartner: Boundary values versus dilatations of harmonic mappings. - J. Anal. Math. 72, 1997, 141-164.

[3] Bshouty, D., and A. Lyzzaik: Uniqueness of harmonic mappings into strictly starlike domains with rectifiable boundary. - Comput. Methods Funct. Theory 8, 2008, 433-446. 
[4] Bshouty, D., A. Lyzzaik, and A. Weitsman: On the boundary behaviour of univalent harmonic mappings onto convex domains. - Comput. Methods Funct. Theory 8, 2008, 261-275.

[5] Cima, J. A., A. L. Matheson, and W. T. Ross: The Cauchy transform. - Math. Surveys Monogr. 125, Amer. Math. Soc., Providence, RI, 2006.

[6] Clunie, J., and T. Sheil-Small: Harmonic univalent functions. - Ann. Acad. Sci. Fenn. Ser. A I Math. 9, 1984, 3-25.

[7] Colwell, P.: Blaschke products. Bounded analytic functions. - Univ. of Michigan Press, Ann Arbor, MI, 1985.

[8] Duren, P.: Harmonic mappings in the plane. - Cambridge Tracts in Math. 156, Cambridge Univ. Press, Cambridge, 2004.

[9] Heinz, E.: On one-to-one harmonic mappings. - Pacific J. Math. 9, 1959, 101-105.

[10] Hengartner, W., and G. Schober: Harmonic mappings with given dilatation. - J. London Math. Soc. (2) 33, 1986, 473-483.

[11] Kalaj, D.: On harmonic diffeomorphisms of the unit disc onto a convex domain. - Complex Var. Theory Appl. 48, 2003, 175-187.

[12] Laugesen, R. S.: Planar harmonic maps with inner and Balschke dilatations. - J. London Math. Soc. (2) 56, 1997, 37-48.

[13] Pommerenke, Ch.: Boundary behaviour of conformal maps. - Grundlehren Math. Wiss., Springer-Verlag, Berlin, 1992.

[14] Protas, D.: On the accumulation of the zeros of a Blaschke product at a boundary point. Proc. Amer. Math. Soc. 34, 1972, 489-496.

[15] Tsuj, M.: Potential theory in modern function theory. Second edition. - Chelsea Publishing Co., New York, 1975.

Received 18 February 2011 